\title{
RESCALING PLANAR HYPERBOLIC SECTORS
}

\author{
MARCY BARGE
}

(Communicated by Kenneth R. Meyer)

\begin{abstract}
Constant rescaling of a planar hyperbolic sector produces a one parameter family of pairwise not locally topologically conjugate sectors if and only if the planar hyperbolic sector is not locally topologically conjugate to a linear hyperbolic sector.
\end{abstract}

\section{INTRODUCTION}

We consider the effects of constant scaling on planar hyperbolic sectors. Shafer et al. [SSW] constructed a $C^{\infty}$ planar hyperbolic sector that is not locally topologically conjugate to a linear hyperbolic sector. It was observed in that paper that constant rescaling of their example yields a one parameter family of pairwise not locally topologically conjugate hyperbolic sectors. A large collection of exotic planar hyperbolic sectors was constructed in [BSW], demonstrating the richness of the local topological conjugacy class structure of such sectors. For these examples the effect of constant rescaling is not so apparent. The main result of this paper is that a planar hyperbolic sector is not locally topologically conjugate to a linear hyperbolic sector if and only if constant rescaling of the sector produces a one parameter family of pairwise not locally topologically conjugate sectors.

\section{Preliminaries}

We will assume that the reader is familiar with the notion of a closed regular hyperbolic sector (cf. Hartman [H]). Such an object will be denoted by $(X, D)$, or simply by $X$, where $X$ is a planar vector field and $D$ is a closed topological disk on which $X$ is a regular hyperbolic sector. We will denote by $H$ the collection of all planar closed regular hyperbolic sectors. As differentiability plays no role in this discussion we require only that $X \in H$ be sufficiently nice as to generate a well-defined (local) flow $\eta_{X}(p, t)$.

We say that $\left(X_{1}, D_{1}\right) \in H$ and $\left(X_{2}, D_{2}\right) \in H$ are locally topologically conjugate if there is a homeomorphism $\psi: U_{1} \rightarrow U_{2}$ of a neighborhood $U_{1}$ of

Received by the editors July 6, 1988 and, in revised form, September 30, 1988.

1980 Mathematics Subject Classification (1985 Revision). Primary 34C10, 34D30; Secondary $58 \mathrm{~F} 10,58 \mathrm{~F} 15$.

Research partially supported by a grant from MONTS. 
the singularity of $X_{1}$ in $D_{1}$ onto a neighborhood $U_{2}$ of the singularity of $X_{2}$ in $D_{2}$ such that $\psi\left(\eta_{X_{1}}(p, t)\right)=\eta_{X_{2}}(\psi(p, t))$ for all $p \in U$, and $t$ such that $\eta_{X_{1}}(p, t) \in U_{1}$.

Associated with each $(X, D) \in H$ are entrance and exit sections $\Sigma_{1}=\Sigma_{1}(X)$ and $\Sigma_{2}=\Sigma_{2}(X)$, respectively, on the boundary of $D$. Let $g:[0,1] \rightarrow \Sigma_{1}$ be a homeomorphism parameterizing $\Sigma_{1}$ with $g(0)$ the intersection of $\Sigma_{1}$ with the stable separatrix of the singularity in $D$ (see Figure 1 ). We define the transit-time map $\tau_{X}$ of $(X, D), \tau_{X}:(0,1] \rightarrow \mathbf{R}^{+}$, by

$$
\tau_{X}(s)=\inf \left\{t \mid \eta_{X}(g(s), t) \in \Sigma_{2}\right\} .
$$

The map $\tau_{X}$ is continuous and $\lim _{s \rightarrow 0+} \tau_{X}(s)=\infty$. Clearly $\tau_{X}$ depends on the choice of parameterization $g$ but the equivalence class of $\tau_{X}$, to be defined momentarily, does not.

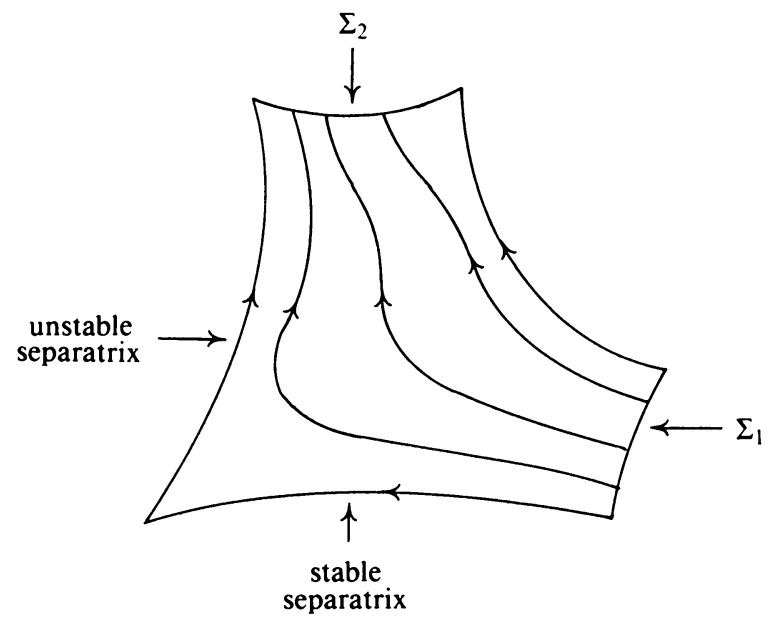

FIGURE 1

Let $T=\left\{\tau:(0,1] \rightarrow \mathbf{R}^{+} \mid \tau\right.$ is continuous and $\left.\lim _{s \rightarrow 0^{+}} \tau(s)=\infty\right\}$. Given $\tau_{1}, \tau_{2} \in T$, we define $\tau_{1} \sim \tau_{2}$ if and only if there is a homeomorphism $h:(0,1] \rightarrow(0,1]$ and a constant $K$ such that $\lim _{s \rightarrow 0^{+}} \tau_{1}(h(s))-\tau_{2}(s)=K$. The relation $\sim$ is then an equivalence relation on $T$ and if $X \in H$, the equivalence class of $\tau_{X}$ in $T$ does not depend on the choice of parameterization of $\Sigma_{1}(X)$ made above.

The following proposition is proved in [SSW].

Proposition 1.1 ([SSW]). If $X_{1}, X_{2} \in H$ then $X_{1}$ and $X_{2}$ are locally topologically conjugate if and only if $\tau_{X_{1}} \sim \tau_{X_{2}}$.

Example. Let $L$ be the planar vector field

$$
L(x, y)=-x \frac{\partial}{\partial x}+y \frac{\partial}{\partial y}
$$

and let $C=\{(x, y) \mid 0 \leq x \leq 2,0 \leq y \leq 2, x y \leq 2\}$. We will call $(L, C) \in H$ the linear hyperbolic sector. If we parameterize $\Sigma_{1}(L)$ by $g(s)=(2, s)$ then $\tau_{L}(s)=\ln (2 / s)$. If $X \in H$ is such that $\tau_{X}$ is monotonic then $X$ and $L$ are locally topologically conjugate. 
A map $\tau \in T$ is called uniformly nonmonotonic provided there is an $a>0$ and a sequence $s_{n}, s_{0}>s_{1}>\cdots$ with $s_{n} \rightarrow 0$, such that $\tau\left(s_{2 n}\right)-\tau\left(s_{2 n+1}\right) \geq a$ for all $n \geq 0$. If $\tau$ is not uniformly nonmonotonic, we will say that $\tau$ is asymptotically monotonic.

Proposition 1.2 ([SSW]). Let $\tau \in T$ then $\tau \sim \tau_{L}$ if and only if $\tau$ is asymptotically monotonic. Thus $X \in H$ is locally topologically conjugate with the linear hyperbolic sector $L$ if and only if $\tau_{X}$ is asymptotically monotonic. [SSW].

An example of a $C^{\infty} X$ with $\tau_{X}$ uniformly nonmonotonic is constructed in

\section{EFFECT OF CONSTANT SCALING ON MEMBERS OF $H$}

Given $(X, D) \in H$ and a positive real number $\lambda$ we may rescale the vector field $X$ by multiplication by $\lambda$ to obtain another hyperbolic sector $(\lambda X, D) \in$ $H$. It is easy to check that $\tau_{\lambda X}=\tau_{X} / \lambda$. For each $\tau \in T$ we will let $G(\tau)=$ $\left\{\lambda \in \mathbf{R}^{+} \mid \lambda \tau \sim \tau\right\} . G(\tau)$ is then a multiplicative subgroup of $\mathbf{R}^{+}$and depends only on the equivalence class of $\tau$ in $T$.

Theorem 2.1. For $\tau \in T, G(\tau)$ is either cyclic or is all of $\mathbf{R}^{+}$. The latter is the case if and only if $\tau \sim \tau_{L}$.

The proof of Theorem 2.1 will be given following the statements and proofs of two corollaries. According to Theorem 2.1, G( $\tau)$ is either $\{1\},\left\{\lambda^{n} \mid n \in Z\right\}$ for some $\lambda>1$, or all of $\mathbf{R}^{+}$. Each of these possibilities can be realized by a transit-time map of a $C^{\infty}$ hyperbolic sector: $G\left(\tau_{X}\right)=\{1\}$ for the hyperbolic sector $X$ constructed in [SSW]; given $\lambda>1$ one can construct a $C^{\infty} X$ with $G\left(\tau_{X}\right)=\langle\lambda\rangle$ using techniques developed in [BSW]; and $G\left(\tau_{L}\right)=\mathbf{R}^{+}$.

Corollary 2.2. Given $X \in H,\left\{\lambda \in \mathbf{R}^{+} \mid X\right.$ is locally topologically conjugate with $\lambda X\}$ is either (multiplicatively) cyclic or is all of $\mathbf{R}^{+}$. The latter is the case if and only if $X$ is locally topologically conjugate with the linear hyperbolic sector $L$.

Proof of Corollary 2.2. This is immediate from Proposition 1.1 and Theorem 2.1.

Corollary 2.3. Suppose $X \in H$ is not locally topologically conjugate with the linear hyperbolic sector $L$. There is then an $\varepsilon>0$ such that $\lambda_{1} X$ and $\lambda_{2} X$ are not locally topologically conjugate provided $\lambda_{1} \neq \lambda_{2}$ and $\left|1-\lambda_{i}\right|<\varepsilon, i=1,2$.

Proof of Corollary 2.3. Theorem 2.1 and Proposition 1.1 imply that there is an $\eta>1$ such that $G\left(\tau_{X}\right)=\left\{\eta^{n} \mid n \in Z\right\}$. Let $\varepsilon=1-\eta^{-1 / 2}$ and let $\lambda_{1}$ and $\lambda_{2}$ satisfy $\lambda_{1}<\lambda_{2}$ and $\left|1-\lambda_{i}\right|<\varepsilon$ for $i=1,2$. Suppose that $\lambda_{1}^{-1} \tau_{X} \sim \lambda_{2}^{-1} \tau_{X}$. Then there is a homeomorphism $h:(0,1] \rightarrow(0,1]$ and a constant $K$ such that

$$
\lim _{s \rightarrow 0^{+}} \lambda_{1}^{-1} \tau_{X}(h(s))-\lambda_{2}^{-1} \tau_{X}(s)=K .
$$


Then

$$
\lim _{s \rightarrow 0^{+}} \tau_{X}\left(h^{-1}(s)\right)-\lambda_{1}^{-1} \lambda_{2} \tau_{X}(s)=-\lambda_{2} K
$$

so that $\lambda_{1}^{-1} \lambda_{2} \in G\left(\tau_{X}\right)$. But

$$
1-\left(1-\eta^{-1 / 2}\right)<\lambda_{1}<\lambda_{2}<1+\left(1-\eta^{-1 / 2}\right)
$$

so that

$$
1<\lambda_{1}^{-1} \lambda_{2}<\eta^{1 / 2}\left(2-\eta^{-1 / 2}\right) .
$$

But $\eta^{1 / 2}\left(2-\eta^{-1 / 2}\right)=2 \eta^{1 / 2}-1<\eta$ since $\eta>1$ so that $1<\lambda_{1}^{-1} \lambda_{2}<\eta$ and $\lambda_{1}^{-1} \lambda_{2}$ cannot be in $G\left(\tau_{X}\right)$. Thus $\lambda_{1}^{-1} \tau_{X}$ and $\lambda_{2}^{-1} \tau_{X}$ are not equivalent and it follows from Proposition 1.1 that $\lambda_{1} X$ and $\lambda_{2} X$ are not locally topologically conjugate.

We turn now to the proof of Theorem 2.1. If $\tau \in T$ and $\tau \not \tau_{L}, \tau(s)$ must have oscillations that don't damp out as $s \rightarrow 0$. The idea of the proof is to show that, corresponding to $\lambda \in G(\tau), \lambda>1$, there must be a regularity in the occurrence of the larger oscillations of $\tau(s)$ as $s \rightarrow 0$. From this regularity we will find that 1 must be isolated in $G(\tau)$; that $G(\tau)$ is cyclic will then follow easily.

Given $\tau \in T$ and $\lambda \in G(\tau)$ we will let $h_{\lambda}:(0,1] \rightarrow(0,1]$ and $K_{\lambda}$ be any homeomorphism and constant, respectively, such that

$$
\lim _{s \rightarrow 0^{+}} \tau\left(h_{\lambda}(s)\right)-\lambda \tau(s)=K_{\lambda} .
$$

Lemma 2.4. Let $\tau \in T$ and suppose that $\lambda \in G(\tau), \lambda>1$. Then $\tau\left(h_{\lambda}^{-1}(s)\right)<$ $\tau(s)$ for all sufficiently small $s \in(0,1]$.

Proof of Lemma 2.4. Let $C<\infty$ be such that $\left|\tau\left(h_{\lambda}(s)\right)-\lambda \tau(s)-K_{\lambda}\right| \leq C$ for all $s$. Since $\lambda>1$ and $\tau\left(h_{\lambda}^{-1}(s)\right) \rightarrow \infty$ as $s \rightarrow 0^{+},(\lambda-1) \tau\left(h_{\lambda}^{-1}(s)\right)>K_{\lambda}+C$ for all sufficiently small $s \in(0,1]$. For $s$ this small we have

$$
\begin{aligned}
\tau(s)=\tau\left(h\left(h_{\lambda}^{-1}(s)\right)\right) & \geq \lambda \tau\left(h_{\lambda}^{-1}(s)\right)-K_{\lambda}-C \\
& >\tau\left(h_{\lambda}^{-1}(s)\right) .
\end{aligned}
$$

Lemma 2.5. Suppose that $\tau \in T, \lambda \in G(\tau)$, and $\lambda>1$. Then for all sufficiently small $s \in(0,1], h_{\lambda}(s)<s$ and $\lim _{n \rightarrow \infty} h_{\lambda}^{n}(s)=0$. Also, given $M \in Z^{+}$, $s<h_{\lambda}^{-1}(s)<\cdots<h_{\lambda}^{-M}(s)$ for all sufficiently small $s \in(0,1]$.

Proof of Lemma 2.5. Let $C<\infty$ be such that $\left|\tau\left(h_{\lambda}(s)\right)-\lambda \tau(s)-K_{\lambda}\right| \leq C$ for all $s \in(0,1]$ and let $s_{n} \in(0,1]$ be a sequence such that $s_{n} \rightarrow 0$ as $n \rightarrow \infty$ and $\tau\left(s_{n}\right)=\max \left\{\tau(s) \mid s_{n} \leq s \leq 1\right\}$. Since $\tau(s) \rightarrow \infty$ as $s \rightarrow 0$ and $\lambda>1$ there is an $N$ such that $(\lambda-1) \tau\left(s_{n}\right)>C-K_{\lambda}$ for all $n \geq N$. Then $\left|\tau\left(h_{\lambda}\left(s_{n}\right)\right)-\lambda \tau\left(s_{n}\right)-K_{\lambda}\right| \leq C$ implies that

$$
\begin{aligned}
\tau\left(h_{\lambda}\left(s_{n}\right)\right) & \geq K_{\lambda}-C+\lambda \tau\left(s_{n}\right) \\
& >K_{\lambda}-C+C-K_{\lambda}+\tau\left(s_{n}\right) \text { for all } n \geq N .
\end{aligned}
$$


Thus $\tau\left(h_{\lambda}\left(s_{n}\right)\right)>\tau\left(s_{n}\right)$ for all $n \geq N$ so that $h_{\lambda}\left(s_{n}\right)<s_{n}$, by choice of $s_{n}$, for all $n \geq N$. It follows from this that if there is a sequence $s_{n}^{\prime}$ in $(0,1]$ with $s_{n}^{\prime} \rightarrow 0$ as $n \rightarrow \infty$ and $h_{\lambda}\left(s_{n}^{\prime}\right) \geq s_{n}^{\prime}$, then there is another sequence $s_{n}^{\prime \prime}$ in $(0,1]$ with $s_{n}^{\prime \prime} \rightarrow 0$ as $n \rightarrow \infty$ and $h_{\lambda}\left(s_{n}^{\prime \prime}\right)=s_{n}^{\prime \prime}$. But, for sufficiently large $n$, $(\lambda-1) \tau\left(s_{n}^{\prime \prime}\right)>K_{\lambda}+C$ so that $\lambda \tau\left(s_{n}^{\prime \prime}\right)-\tau\left(h_{\lambda}\left(s_{n}^{\prime \prime}\right)\right)>K_{\lambda}+C$ and this is inconsistent with $\left|\tau\left(h_{\lambda}\left(s_{n}^{\prime \prime}\right)\right)-\lambda \tau\left(s_{n}^{\prime \prime}\right)-K_{\lambda}\right| \leq C$. Thus, for all sufficiently small $s, h_{\lambda}(s)<s$. If, for such $s, h_{\lambda}^{n}(s) \nrightarrow 0$ then $h_{\lambda}^{n}(s) \rightarrow s^{\prime}>0$ with $h_{\lambda}\left(s^{\prime}\right)=s^{\prime}<s$ but this doesn't happen for small enough $s$.

If $S \in(0,1]$ is such that $h_{\lambda}(s)<s$ for all $s \leq S$ then $s<h_{\lambda}^{-1}(s)<\cdots<$ $h_{\lambda}^{-M}(s)$ for all $s \leq h_{\lambda}^{M}(S)$.

Lemma 2.6. Let $\tau \in T$ and suppose that $\lambda_{1}, \lambda_{2} \in G(\tau)$ and $M \in Z^{+}$are such that $1<\lambda_{1}^{M}<\lambda_{2}$. Then

$$
h_{\lambda_{2}}(s)<h_{\lambda_{1}}^{-1}\left(h_{\lambda_{2}}(s)\right)<h_{\lambda_{1}}^{-2}\left(h_{\lambda_{2}}(s)\right)<\cdots<h_{\lambda_{1}}^{-M}\left(h_{\lambda_{2}}(s)\right)<s
$$

for all sufficiently small $s \in(0,1]$.

Proof of Lemma 2.6. We need only check that $h_{\lambda_{1}}^{-M}\left(h_{\lambda_{2}}(s)\right)<s$ for sufficiently small $s$. As $s \rightarrow 0^{+}$we have

$$
\tau\left(h_{\lambda_{1}}(s)\right)-\lambda_{1} \tau(s) \rightarrow K_{\lambda_{1}}
$$

so that

$$
\lambda_{1}^{-1} \tau(s)-\tau\left(h_{\lambda_{1}}^{-1}(s)\right) \rightarrow \lambda_{1}^{-1} K_{\lambda_{1}}
$$

and

$$
\lambda_{1}^{-1} \tau\left(h_{\lambda_{1}}^{-1}(s)\right)-\tau\left(h_{\lambda_{1}}^{-2}(s)\right) \rightarrow \lambda_{1}^{-1} K_{\lambda_{1}}
$$

Thus

so that

$$
\lambda_{1}^{-1}\left[\lambda_{1}^{-1} \tau(s)-\lambda_{1}^{-1} K_{\lambda_{1}}\right]-\tau\left(h_{\lambda_{1}}^{-2}(s)\right) \rightarrow \lambda_{1}^{-1} K_{\lambda_{1}}
$$

$$
\tau\left(h_{\lambda_{1}}^{-2}(s)\right)-\lambda_{1}^{-2} \tau(s) \rightarrow-2 \lambda_{1}^{-1} K_{\lambda_{1}} \quad \text { as } s \rightarrow 0^{+} .
$$

Continuing in this way we get

$$
\tau\left(h_{\lambda_{1}}^{-M}(s)\right)-\lambda_{1}^{-M} \tau(s) \rightarrow-M \lambda_{1}^{-1} K_{\lambda_{1}} \text { as } s \rightarrow 0^{+} .
$$

Since $\tau\left(h_{\lambda_{2}}(s)\right)-\lambda_{2} \tau(s) \rightarrow K_{\lambda_{2}}$ and

$$
\tau\left(h_{\lambda_{1}}^{-M}\left(h_{\lambda_{2}}(s)\right)\right)-\lambda_{1}^{-M} \tau\left(h_{\lambda_{2}}(s)\right) \rightarrow-M \lambda_{1}^{-1} K_{\lambda_{1}} \text { as } s \rightarrow 0^{+},
$$

we have

$$
\tau\left(h_{\lambda_{1}}^{-M}\left(h_{\lambda_{2}}(s)\right)\right)-\lambda_{1}^{-M} \lambda_{2} \tau(s) \rightarrow C=\lambda_{1}^{-M} K_{\lambda_{2}}-M \lambda_{1}^{-1} K_{\lambda_{1}} \quad \text { as } s \rightarrow 0^{+} .
$$

Since $\lambda_{1}^{-M} \lambda_{2}<1$, it follows from this with the argument in the proof of Lemma 2.5 that $h_{\lambda_{1}}^{-M}\left(h_{\lambda_{2}}(s)\right)<s$ for all sufficiently small $s \in(0,1]$. 
Lemma 2.7. Let $\tau \in T$ and suppose that $\lambda \in G(\tau)$ with $\lambda>1$. Given $\varepsilon>0$ let $S \in(0,1]$ be small enough so that $\left|\tau\left(h_{\lambda}(s)\right)-\tau(s)-K_{\lambda}\right| \leq \varepsilon / 2$ and $h_{\lambda}(s)<s$ for all $s \leq S$. Then, for any $s_{1}, s_{2} \in(0, S]$,

$$
\begin{aligned}
\lambda^{n} \mid \tau\left(s_{1}\right) & -\tau\left(s_{2}\right) \mid-\varepsilon\left(\lambda^{n}\right)(\lambda-1)^{-1} \\
& \leq\left|\tau\left(h_{\lambda}^{n}\left(s_{1}\right)\right)-\tau\left(h_{\lambda}^{n}\left(s_{2}\right)\right)\right| \\
& \leq \lambda^{n}\left|\tau\left(s_{1}\right)-\tau\left(s_{2}\right)\right|+\varepsilon\left(\lambda^{n}-1\right)(\lambda-1)^{-1}
\end{aligned}
$$

for all $n>0$. Also, given $M \in Z^{+}, S$ can be taken small enough so that, for any $s_{1}, s_{2} \in(0, S]$,

$$
\left|\tau\left(h_{\lambda}^{-k}\left(s_{1}\right)\right)-\tau\left(h_{\lambda}^{-k}\left(s_{2}\right)\right)\right| \geq \lambda^{-M}\left|\tau\left(s_{1}\right)-\tau\left(s_{2}\right)\right|-\varepsilon\left(\lambda^{-M}-1\right)\left(\lambda^{-1}-1\right)^{-1}
$$

for $k=0,1, \ldots, M$.

Proof of Lemma 2.7. The first two inequalities are correct for $n=0$. Suppose that

$$
\left|\tau\left(h_{\lambda}^{n}\left(s_{1}\right)\right)-\tau\left(h_{\lambda}^{n}\left(s_{2}\right)\right)\right| \leq \lambda^{n}\left|\tau\left(s_{1}\right)-\tau\left(s_{2}\right)\right|+\varepsilon\left(\lambda^{n}-1\right)(\lambda-1)^{-1}
$$

for some $n$. Then

$$
\begin{aligned}
\left|\tau\left(h_{\lambda}^{n+1}\left(s_{1}\right)\right)-\tau\left(h_{\lambda}^{n+1}\left(s_{2}\right)\right)\right| \leq & \mid \tau\left(h_{\lambda}\left(h_{\lambda}^{n}\left(s_{1}\right)\right)\right)-\lambda \tau\left(h_{\lambda}^{n}\left(s_{1}\right)\right)-K_{\lambda}+\lambda \tau\left(h_{\lambda}^{n}\left(s_{1}\right)\right) \\
& -\left[\tau\left(h_{\lambda}\left(h_{\lambda}^{n}\left(s_{2}\right)\right)\right)-\lambda \tau\left(h_{\lambda}^{n}\left(s_{2}\right)\right)-K_{\lambda}+\lambda \tau\left(h_{\lambda}^{n}\left(s_{2}\right)\right)\right] \mid \\
\leq & \frac{\varepsilon}{2}+\frac{\varepsilon}{2}+\lambda\left|\tau\left(h_{\lambda}^{n}\left(s_{1}\right)\right)-\tau\left(h_{\lambda}^{n}\left(s_{2}\right)\right)\right| \\
\leq & \varepsilon+\lambda\left[\lambda^{n}\left|\tau\left(s_{1}\right)-\tau\left(s_{2}\right)\right|+\varepsilon\left(\lambda^{n}-1\right)(\lambda-1)^{-1}\right] \\
\leq & \lambda^{n+1}\left|\tau\left(s_{1}\right)-\tau\left(s_{2}\right)\right|+\varepsilon\left[\lambda\left(\lambda^{n}-1\right)(\lambda-1)^{-1}+1\right] \\
\leq & \lambda^{n+1}\left|\tau\left(s_{1}\right)-\tau\left(s_{2}\right)\right|+\varepsilon\left(\lambda^{n+1}-1\right)(\lambda-1)^{-1},
\end{aligned}
$$

and the second inequality in the lemma is established by induction. The other conclusions can be obtained in a similar manner.

Now suppose that $\tau \in T, J$ is a closed subinterval of $(0,1]$, and $r>0$. Let $M(J, r)$ be defined by $M(J, r)=\max \left\{n \mid\right.$ there exist $s_{1}, s_{2}, \ldots, s_{n} \in J$ with $s_{1}<s_{2}<\cdots<s_{n}$ and $(-1)^{k}\left(\tau\left(s_{k+1}\right)-\tau\left(s_{k}\right)\right) \geq r$ for $\left.k=1, \ldots, n-1\right\}$. Thus $M(J, r)-1$ is the maximum number of oscillations of size at least $r$ for $\left.\tau\right|_{J}$.

Lemma 2.8. Let $\tau \in T$ and suppose that $\lambda \in G(\tau)$ with $\lambda>1$. Let $\varepsilon>0$ be given and let $S \in(0,1]$ be small enough so that $h_{\lambda}(s)<s$ and $\mid \tau\left(h_{\lambda}(s)\right)-\tau(s)-$ $K_{\lambda} \mid \leq \varepsilon / 2$ for all $s \in(0, S]$. Then

$$
M\left(\left[h_{\lambda}^{n+1}(s), h_{\lambda}^{n}(s)\right], \lambda^{n} a+\varepsilon\left(\lambda^{n}-1\right)(\lambda-1)^{-1}\right) \leq M\left(\left[h_{\lambda}(s), s\right], a\right)
$$

for all $a>0, s \in(0, S]$, and $n \geq 0$. 
Proof of Lemma 2.8. Suppose that $n \geq 1$ and that $\left\{s_{1}, s_{2}, \ldots, s_{m}\right\} \subseteq\left[h_{\lambda}^{n+1}(s)\right.$, $\left.h_{\lambda}^{n}(s)\right]$ satisfy $s_{1}<s_{2}<\cdots<s_{m}$ and $(-1)^{k}\left(\tau\left(s_{k+1}\right)-\tau\left(s_{k}\right)\right) \geq \lambda^{n} a+\varepsilon\left(\lambda^{n}-1\right)$ $(\lambda-1)^{-1}$ for $k=1, \ldots, m-1$. Then $\left\{h_{\lambda}^{-1}\left(s_{1}\right), \ldots, h_{\lambda}^{-1}\left(s_{m}\right)\right\} \subseteq\left[h_{\lambda}^{n}(s), h_{\lambda}^{n-1}(s)\right]$ and $h_{\lambda}^{-1}\left(s_{1}\right)<h_{\lambda}^{-1}\left(s_{2}\right)<\cdots<h_{\lambda}^{-1}\left(s_{m}\right)$. Also,

$$
\begin{aligned}
\tau\left(s_{k+1}\right)-\tau\left(s_{k}\right)= & \tau\left(h_{\lambda}\left(h_{\lambda}^{-1}\left(s_{k+1}\right)\right)\right)-\tau\left(h_{\lambda}\left(h_{\lambda}^{-1}\left(s_{k}\right)\right)\right) \\
= & \tau\left(h_{\lambda}\left(h_{\lambda}^{-1}\left(s_{k+1}\right)\right)\right)-\lambda \tau\left(h_{\lambda}^{-1}\left(s_{k+1}\right)\right)-K_{\lambda} \\
& -\left(\tau\left(h_{\lambda}\left(h_{\lambda}^{-1}\left(s_{k}\right)\right)\right)-\lambda \tau\left(h_{\lambda}^{-1}\left(s_{k}\right)\right)-K_{\lambda}\right) \\
& +\lambda \tau\left(h_{\lambda}^{-1}\left(s_{k+1}\right)\right)-\lambda \tau\left(h_{\lambda}^{-1}\left(s_{k}\right)\right) .
\end{aligned}
$$

From

$$
\left|\tau\left(h_{\lambda}\left(h_{\lambda}^{-1}(s)\right)\right)-\tau\left(h_{\lambda}^{-1}(s)\right)-K_{\lambda}\right| \leq \varepsilon / 2
$$

and the above we get

$$
\begin{aligned}
-\varepsilon & +\lambda\left(\tau\left(h_{\lambda}^{-1}\left(s_{k+1}\right)\right)-\tau\left(h_{\lambda}^{-1}\left(s_{k}\right)\right)\right) \\
& \leq \tau\left(s_{k+1}\right)-\tau\left(s_{k}\right) \leq \varepsilon+\lambda\left(\tau\left(h_{\lambda}^{-1}\left(s_{k+1}\right)\right)-\tau\left(h_{\lambda}^{-1}\left(s_{k}\right)\right)\right) .
\end{aligned}
$$

Thus

$$
(-1)^{k}\left(\tau\left(s_{k+1}\right)-\tau\left(s_{k}\right)\right) \leq \varepsilon+\lambda(-1)^{k}\left(\tau\left(h_{\lambda}^{-1}\left(s_{k+1}\right)\right)-\tau\left(h_{\lambda}^{-1}\left(s_{k}\right)\right)\right)
$$

and we have

$$
\begin{aligned}
& \varepsilon+\lambda(-1)^{k}\left(\tau\left(h_{\lambda}^{-1}\left(s_{k+1}\right)\right)-\tau\left(h_{\lambda}^{-1}\left(s_{k}\right)\right)\right) \\
& \quad \geq \lambda^{n} a+\varepsilon\left(\lambda^{n}-1\right)(\lambda-1)^{-1} \text { for } k=1, \ldots, m-1 .
\end{aligned}
$$

It follows that

$$
(-1)^{k}\left(\tau\left(h_{\lambda}^{-1}\left(s_{k+1}\right)\right)-\tau\left(h_{\lambda}^{-1}\left(s_{k}\right)\right)\right) \geq \lambda^{n-1} a+\varepsilon\left(\lambda^{n-1}-1\right)(\lambda-1)^{-1}
$$

for $k=1, \ldots, m-1$ so that

$$
\begin{gathered}
M\left(\left[h_{\lambda}^{n}(s), h_{\lambda}^{n-1}(s)\right], \lambda^{n-1} a+\varepsilon\left(\lambda^{n-1}-1\right)(\lambda-1)^{-1}\right) \\
\quad \geq M\left(\left[h_{\lambda}^{n+1}(s), h_{\lambda}^{n}(s)\right], \lambda^{n} a+\varepsilon\left(\lambda^{n}-1\right)(\lambda-1)^{-1}\right)
\end{gathered}
$$

for all $n \geq 1$.

Lemma 2.9. Let $\tau \in T$ and suppose that $\tau$ is uniformly nonmonotonic (see $\S 1$ ). If there is a $\lambda_{2} \in G(\tau)$ with $\lambda_{2}>1$ then there is an $M \in Z^{+}$such that $\lambda \notin G(\tau)$ for any $\lambda$ satisfying $1<\lambda<\lambda_{2}^{1 / M}$.

Proof of Lemma 2.9. Let $a>0$ be such that $\tau\left(t_{2 n}\right)-\tau\left(t_{2 n+1}\right) \geq a$ for all $n$ where $t_{0}<t_{1}<\cdots$ is a sequence in $(0,1]$ with $t_{n} \rightarrow 0$ as $n \rightarrow \infty$. Let $\lambda_{2}$ be in $G(\tau)$ with $\lambda_{2}>1$ and let $\varepsilon=\left(\left(\lambda_{2}-1\right) /\left(\lambda_{2}+1\right)^{-1} \frac{a}{4}\right)$. There is then an $m$ large enough so that $h_{\lambda_{2}}(s)<s$ and $\left|\tau\left(h_{\lambda_{2}}(s)\right)-\lambda_{2} \tau(s)-K_{\lambda_{2}}\right| \leq \varepsilon / 2$ for all $s \leq t_{2 m}$. Let $t_{2 m}=t^{\prime}$ and $t_{2 m+1}=t^{\prime \prime}$ so that $t^{\prime \prime}<t^{\prime}$ and $\tau\left(t^{\prime}\right)-\tau\left(t^{\prime \prime}\right) \geq a$. 
Let $M=M\left(\left[h_{\lambda_{2}}\left(t^{\prime}\right), t^{\prime}\right], a / 2\right)$ and suppose there is a $\lambda_{1} \in G(\tau)$ such that $1<\lambda_{1}^{M}<\lambda_{2}$. From Lemma 2.8 we have

$$
M\left(\left[h_{\lambda_{2}}^{n+1}\left(t^{\prime}\right), h_{\lambda_{2}}^{n}\left(t^{\prime}\right)\right],\left(\frac{a}{2}\right) \lambda_{2}^{n}+\varepsilon\left(\lambda_{2}^{n}-1\right)\left(\lambda_{2}-1\right)^{-1}\right) \leq M
$$

for all $n \in Z^{+}$. Also, from Lemma 2.7,

$$
\tau\left(h_{\lambda_{2}}^{n+1}\left(t^{\prime}\right)\right)-\tau\left(h_{\lambda_{2}}^{n+1}\left(t^{\prime \prime}\right)\right) \geq a \lambda_{2}^{n+1}-\varepsilon\left(\lambda_{2}^{n+1}-1\right)\left(\lambda_{2}-1\right)^{-1}
$$

for all $n \in Z^{+}$.

Let $s_{1}=h_{\lambda_{2}}^{n+1}\left(t^{\prime}\right)$ and let $s_{0}=\sup \left\{s \mid s<s_{1}, \tau(s)=\tau\left(h_{\lambda_{2}}^{n+1}\left(t^{\prime \prime}\right)\right)\right\}$. Then $s_{0}<s_{1}$ and, from (2),

$$
\tau\left(s_{1}\right)-\tau\left(s_{0}\right) \geq a \lambda_{2}^{n+1}-\varepsilon\left(\lambda_{2}^{n+1}-1\right)\left(\lambda_{2}-1\right)^{-1} .
$$

Now, $s_{0}$ and $s_{1}$ depend on $n$ and go to 0 as $n$ goes to $\infty$ (Lemma 2.5). It follows from Lemmas 2.4 and 2.5 that, for $n$ sufficiently large,

$$
\tau\left(h_{\lambda_{1}}^{-1}(s)\right)<\tau(s) \text { and } h_{\lambda_{1}}^{-1}(s)>s
$$

for all $s<h_{\lambda_{2}}^{n}\left(t^{\prime}\right)$. We see then, from the definition of $s_{0}$, that $h_{\lambda_{1}}^{-1}\left(s_{0}\right)>s_{1}$. Now let $s_{2 k}=h_{\lambda_{1}}^{-k}\left(s_{0}\right)$ for $k=1,2, \ldots, M$ and $s_{2 k+1}=h_{\lambda_{1}}^{-k}\left(s_{1}\right)$ for $k=$ $0,1, \ldots, M$. From Lemma 2.5 we have, for $n$ sufficiently large,

$$
s_{1}<s_{2}<\cdots<s_{2 M+1} \text { and } s_{i} \in\left[h_{\lambda_{2}}^{n+1}\left(t^{\prime}\right), h_{\lambda_{2}}^{n}\left(t^{\prime}\right)\right]
$$

for $i=1, \ldots, 2 M+1$.

We will show now, that for sufficiently large $n$,

$$
(-1)^{i}\left(\tau\left(s_{i+1}\right)-\tau\left(s_{i}\right)\right) \geq(a / 2) \lambda_{2}^{n}+\varepsilon\left(\lambda_{2}^{n}-1\right)\left(\lambda_{2}-1\right)^{-1}
$$

for $i=1, \ldots, 2 M$. Let $N$ be large enough so that (4) and (5) hold for all $n \geq N$ and so that $\tau\left(h_{\lambda_{1}}^{-1}(s)\right)<\tau(s)$ for all $s \in\left[h_{\lambda_{2}}^{n+1}\left(t^{\prime}\right), h_{\lambda_{2}}^{n}\left(t^{\prime}\right)\right]$ and all $n \geq N$ (Lemma 2.5).

From (3), (4), (5), and the definition of the $s_{k}$ we have

$$
(-1)\left(\tau\left(s_{2 k+2}\right)-\tau\left(s_{2 k+1}\right)\right) \geq \tau\left(s_{2 k+1}\right)-\tau\left(s_{2 k}\right)
$$

for $k=0,1, \ldots, M-1$ and

$$
(-1)\left(\tau\left(s_{2}\right)-\tau\left(s_{1}\right)\right) \geq a \lambda_{2}^{n+1}-\varepsilon\left(\lambda_{2}^{n+1}-1\right)\left(\lambda_{2}-1\right)^{-1}
$$

for all $n \geq N$.

Let $\delta_{n}=\frac{1}{2} \inf \left\{\left|\tau\left(h_{\lambda_{1}}(s)\right)-\lambda_{1} \tau(s)-K_{\lambda_{1}}\right|: s \leq h_{\lambda_{2}}^{n}\left(t^{\prime}\right)\right\}$. Then $\delta_{n} \rightarrow 0$ as $n \rightarrow \infty$ and, from Lemma 2.5 and (7), we have

$$
\begin{aligned}
\tau\left(s_{2 k+1}\right) & -\tau\left(s_{2 k}\right) \geq \lambda_{1}^{-M}\left[\tau\left(s_{1}\right)-\tau\left(s_{0}\right)\right]-\delta_{n}\left(\lambda_{1}^{-M}-1\right)\left(\lambda_{1}^{-1}-1\right)^{-1} \\
& \geq \lambda_{1}^{-M}\left[a \lambda_{2}^{n+1}-\varepsilon\left(\lambda_{2}^{n+1}-1\right)\left(\lambda_{2}-1\right)^{-1}\right]-\delta_{n}\left(\lambda_{1}^{-M}-1\right)\left(\lambda_{1}^{-1}-1\right)^{-1}
\end{aligned}
$$

for $k=0,1, \ldots, M$ and $n \geq N$. 
From (7) and (8) we obtain

$$
\begin{aligned}
(-1)^{k}\left(\tau\left(s_{k+1}\right)-\tau\left(s_{k}\right)\right) \geq & \lambda_{1}^{-M}\left[a \lambda_{2}^{n+1}-\varepsilon\left(\lambda_{2}^{n+1}-1\right)\left(\lambda_{2}-1\right)^{-1}\right] \\
& -\delta_{n}\left(\lambda_{1}^{-M}-1\right)\left(\lambda_{1}^{-1}-1\right)^{-1}
\end{aligned}
$$

for $k=1, \ldots, 2 M$ and all $n \geq N$.

Also, for $n \geq N$ :

$$
\begin{aligned}
\frac{3 a}{4} & \lambda_{2}^{n}\left(\lambda_{1}^{-M} \lambda_{2}-1\right) \\
\leq & a \lambda_{2}^{n}\left[\lambda_{1}^{-M} \lambda_{2}\left(1-\left(\frac{1}{4}\right)\left(1-\lambda_{2}^{-(n+1)}\right)\left(1+\lambda_{2}\right)^{-1}\right)-\frac{1}{2}-\left(\frac{1}{4}\right)\left(1-\lambda_{2}^{-n}\right)\left(1+\lambda_{2}\right)^{-1}\right] \\
\leq & \lambda_{1}^{-M}\left[a \lambda_{2}^{n+1}-\left(\left(\lambda_{2}-1\right) /\left(\lambda_{2}+1\right)^{-1}\right)\left(\frac{a}{4}\right)\left(\lambda_{2}^{n+1}-1\right)\left(\lambda_{2}-1\right)^{-1}\right] \\
& -\left(\frac{a}{2}\right) \lambda_{2}^{n}-\left(\left(\lambda_{2}-1\right) /\left(\lambda_{2}+1\right)^{-1}\right)\left(\frac{a}{4}\right)\left(\lambda_{2}^{n}-1\right)\left(\lambda_{2}-1\right)^{-1} \\
\leq & \lambda_{1}^{-M}\left[a \lambda_{2}^{n+1}-\varepsilon\left(\lambda_{2}^{n+1}-1\right)\left(\lambda_{2}-1\right)^{-1}\right] \\
& -\left[\left(\frac{a}{2}\right) \lambda_{2}^{n}+\varepsilon\left(\lambda_{2}^{n}-1\right)\left(\lambda_{2}-1\right)^{-1}\right] .
\end{aligned}
$$

Since $\lambda_{1}^{-M} \lambda_{2}>1, \lambda_{2}>1$, and $\delta_{n} \rightarrow 0$ as $n \rightarrow \infty$, it follows that, for sufficiently large $n$,

$$
\begin{aligned}
\lambda_{1}^{-M}\left[a \lambda_{2}^{n+1}\right. & \left.-\varepsilon\left(\lambda_{2}^{n+1}-1\right)\left(\lambda_{2}-1\right)^{-1}\right]-\delta_{n}\left(\lambda_{1}^{-M}-1\right)\left(\lambda_{1}^{-1}-1\right)^{-1} \\
& \geq(a / 2) \lambda_{2}^{n}+\varepsilon\left(\lambda_{2}^{n}-1\right)\left(\lambda_{2}-1\right)^{-1} .
\end{aligned}
$$

Inequalities (9) and (10) establish the validity of (6) for sufficiently large $n$.

But now $M\left(\left[h_{\lambda_{2}}^{n+1}\left(t^{\prime}\right), h_{\lambda_{2}}^{n}\left(t^{\prime}\right)\right],\left(\frac{a}{2}\right) \lambda_{2}^{n}+\varepsilon\left(\lambda_{2}^{n}-1\right)\left(\lambda_{2}-1\right)^{-1}\right) \geq 2 M+1$ for sufficiently large $n$ in contradiction to (1). Thus $\lambda_{1} \notin G(\tau)$ provided $1<\lambda_{1}^{M}<$ $\lambda_{2}$.

Proof of Theorem 2.1. Suppose that $\tau \in T$ and $\tau \not \tau_{L}$. Then, by Proposition $1.2, \tau$ is uniformly nonmonotonic. If $G(\tau) \neq\{1\}$ then there is a $\lambda_{2} \in G(\tau)$ with $\lambda_{2}>1$. By Lemma 2.9 there is an $M \in Z^{+}$such that $\left(1, \lambda_{2}^{1 / M}\right) \cap G(\tau)=\varnothing$. Since $G(\tau)$ is a subgroup of $\mathbf{R}^{+}, G(\tau)$ must be cyclic (in fact, $G(\tau)=\langle\eta\rangle$, $\eta=\inf \{\lambda \in G(\tau) \mid \lambda>1\})$.

On the other hand, if $\tau \sim \tau_{L}$ then $\lambda \tau \sim \lambda \tau_{L} \sim \tau_{L} \sim \tau$ for all $\lambda \in \mathbf{R}^{+}$so that $G(\tau)=\mathbf{R}^{+}$.

\section{ACKNOWLEDGMENTS}

The author would like to thank Dick Gillette, Richard Swanson, and Russ Walker for helpful discussions.

\section{REFERENCES}

[BSW] M. Barge, R. Swanson, and R. Walker, Conjugacy class structure of smooth hyperbolic sectors (preprint).

[H] P. Hartman, Ordinary differential equations, 2nd ed., Birkhauser, Boston, 1982. 
[SSW] D. Shafer, R. Swanson, and R. Walker, Topological conjugacy of singularities of vector fields, Topology 25 (1986), 521-539.

Department of Mathematical Sciences, Montana State University, Bozeman, MonTANa 59717 